\title{
A Multifunctional Wearable Device with a Graphene/Silver Nanowire Nanocomposite for Highly Sensitive Strain Sensing and Drug Delivery
}

\author{
Ge Shi ${ }^{1}$, Tianqing Liu ${ }^{2}$, Zlatko Kopecki ${ }^{3}$, Allison Cowin ${ }^{3}$, Ivan Lee ${ }^{4}$, Jing-Hong Pai ${ }^{5}$, \\ Sean E. Lowe ${ }^{1}$ and Yu Lin Zhong ${ }^{1, *(1)}$ \\ 1 Centre for Clean Environment and Energy, School of Environment and Science, Gold Coast Campus, \\ Griffith University, Gold Coast, QLD 4222, Australia; ge.shi@griffith.edu.au (G.S.); \\ sean.lowe@griffithuni.edu.au (S.E.L.) \\ 2 QIMR Berghofer Medical Research Institute, Brisbane City, QLD 4006, Australia; \\ michelle.liu@qimrberghofer.edu.au \\ 3 Future Industries Institute, University of South Australia, Mawson Lakes, SA 5095, Australia; \\ zlatko.kopecki@unisa.edu.au (Z.K.); allison.cowin@unisa.edu.au (A.C.) \\ 4 School of Information Technology and Mathematical Sciences, University of South Australia, \\ Adelaide, SA 5001, Australia; ivan.lee@unisa.edu.au \\ 5 Australian National Fabrication Facility-South Australian Node, Future Industries Institute, \\ University of South Australia, Mawson Lakes, SA 5095, Australia; jing-hong.pai@unisa.edu.au \\ * Correspondence: y.zhong@griffith.edu.au; Tel.: +61-7-5552-8209
}

Received: 9 March 2019; Accepted: 28 March 2019; Published: 4 April 2019

\begin{abstract}
Advances in wearable, highly sensitive and multifunctional strain sensors open up new opportunities for the development of wearable human interface devices for various applications such as health monitoring, smart robotics and wearable therapy. Herein, we present a simple and cost-effective method to fabricate a multifunctional strain sensor consisting of a skin-mountable dry adhesive substrate, a robust sensing component and a transdermal drug delivery system. The sensor has high piezoresisitivity to monitor real-time signals from finger bending to ulnar pulse. A transdermal drug delivery system consisting of polylactic-co-glycolic acid nanoparticles and a chitosan matrix is integrated into the sensor and is able to release the nanoparticles into the stratum corneum at a depth of $\sim 60 \mu \mathrm{m}$. Our approach to the design of multifunctional strain sensors will lead to the development of cost-effective and well-integrated multifunctional wearable devices.
\end{abstract}

Keywords: graphene; silver nanowire; multifunctional; wearable; strain sensor; drug delivery

\section{Introduction}

Wearable electronic devices have attracted considerable attention in recent years [1-3]. Specifically, skin-mountable strain sensors have potential applications in personal health monitoring, human motion detection and smart human-machine interaction [4-6]. These sensors often consist of two components: a stimuli-responsive component and a stretchable substrate. The stimuli-responsive component should possess high durability, sensitivity and stretchability, while the substrate should be capable of forming robust and conformal interactions with biological skin. However, there are still many areas for improvement that should be addressed to achieve widespread utilization of wearable devices. For skin-mountable devices, substrates employing traditional medical tapes or elastic films generally have limited stretchability and usage times $[7,8]$. In contrast, a promising alternative is represented by dry adhesive substrates with a fibrillary hierarchical structure [9]. These dry adhesives provide large contact areas with the possibility of repeated adhesion. Similar to 
the development of mobile devices where increasing functionalities are being integrated into single devices, multifunctional wearable devices $[2,10,11]$ open new tech logical possibilities in areas such as human-machine interaction, health monitoring and drug delivery [12]. For example, a single device might be fabricated which can simultaneously deliver drugs and monitor the physiological response. Despite the clear promise of such multifunctional wearable devices, further improvements related to the fabrication process and cost-effectiveness are needed for their adoption.

In terms of materials selection, there have been various approaches utilizing nanomaterials (e.g., silver nanowires [13], carbon nanotubes [14] and chemical vapor deposition (CVD) graphene [15]) as sensing components and elastomeric polymers or textiles as flexible and stretchable substrates $[16,17]$. Graphene nanoplatelets ( $\mathrm{GnPs}$ ) have been widely used in various polymer nanocomposites because of their superior electrical and mechanical properties [18-20]. Each GnP is typically $2-5 \mathrm{~nm}$ in thickness and may contain 3-10 graphene layers [21]. Upon reaching the percolation threshold in a matrix, the individual $\mathrm{GnP}$ forms a physically connected network where the overall resistance of the nanocomposites becomes limited by the interlayer contact resistance of the GnPs [4,22]. A strategy to reduce the contact resistance is to combine GnPs with silver nanowires (AgNWs). AgNWs employed in this work are around $40 \mathrm{~nm}$ in diameter and 20-60 $\mu \mathrm{m}$ in length. Under low strain, nanowires might change wave amplitudes and slide across each other, thus facilitating and maintaining a conducting network to accommodate the strain [23].

Transdermal drug delivery is a promising drug delivery strategy to complement the limitations of traditional oral- and injectable-based methods. Transdermal drug delivery might potentially realize convenient and painless drug delivery and a sustained release profile with reduced side effects [24]. Advances in nanotechnology have led to the development of drug nanocarriers which can pass through the skin and target specific skin layers [25]. Polymers, and in particular hydrogels, can be loaded with drugs for transdermal drug delivery. A hydrogel is a physically or chemically cross-linked 3D polymer network, which is typically swollen with water. Hydrogels provide a relatively safe mechanical and chemical environment for loaded drugs. Applying external stimuli, such as pressure strain, can deform hydrogels, accelerate the hydrogel dissolution and thus control the nanocarrier release rate [26]. Integration of the stimuli-responsive component, dry adhesive substrate and nanocarrier delivery system can realize on-time health monitoring, such as of pulse beat rate, and targeted sustained drug release.

In this study, a low-cost, multifunctional (strain sensing and drug delivery) wearable device was developed on an elastic dry-adhesive substrate. The significance of this study includes using a micropillar film as a substrate to form a robust and conformal interaction with the skin, using a GnP and AgNW composite as a sensing material to generate reproducible sensitivity to strain and integrating a transdermal drug delivery system into the strain sensor. The substrate is a polydimethylsiloxane (PDMS) film with micron-size pillars array which exhibited a pull-off strength of up to $\sim 1.0 \mathrm{~N} \mathrm{~cm}^{-2}$. GnPs and AgNWs in solution are deposited layer by layer through vacuum filtration to form a highly sensitive and robust sandwich structure as the sensing component. In addition, the transdermal drug delivery system consisting of a drug-loaded chitosan hydrogel is able to release drug nanocarriers into the stratum corneum at a depth of $\sim 60 \mu \mathrm{m}$. The multifunctional strain sensor exhibited robust mechanical attachment to the skin, reproducible sensitivity to human motions and effective transdermal drug delivery.

\section{Materials and Methods}

\subsection{Materials}

GnPs were prepared on the basis of a reported procedure [27]. In brief, $0.1 \mathrm{~g}$ of a graphite intercalation compound (GIC, Asbury 1721, Asbury, NJ, USA) was placed in a crucible and transferred to a preheated furnace at $700{ }^{\circ} \mathrm{C}$ for $1 \mathrm{~min}$ for expansion. The worm-like expanded graphite was dispersed in acetone via $1 \mathrm{~h}$ sonication in an ultrasonic bath to produce thinner GnPs. Silver nanowires (AgNW-40) with diameter of $40 \mathrm{~nm}$ and length of 20-60 $\mu \mathrm{m}$ suspended in water were purchased 
from ACS MATERIAL, Pasadena, CA, USA. Deferoxamine, chitosan, acetic acid, polyvinyl alcohol, methylene chloride, Triton X, paraformaldehyde, and 4',6-diamidino-s-phenylindole were purchased from Sigma-Aldrich (Sigma-Aldrich Pty Ltd, Castle Hill, NSW, Australia) and used as received. Transwells ${ }^{\circledR}$ plates were purchased from Corning. Polylactic-co-glycolic acid polymers with fluorescent AlexaFluor (AF488) tag (PolySciTech, West Lafayette, IN, USA) and ProLong Gold mounting medium were purchased from Thermo Fisher Scientific.

\subsection{Fabrication of SU-8 Template}

An oxygen plasma cleaned silicon wafer was first baked at $150{ }^{\circ} \mathrm{C}$ for $10 \mathrm{~min}$ on a hotplate to remove water. Bisphenol A Novolac epoxy (SU-8 3010, MicroChem Corp., Westborough, MA, USA) was spin-coated on the wafer (2000 rpm, $30 \mathrm{~s}$ ), followed by softbaking for $5 \mathrm{~min}$ at $150{ }^{\circ} \mathrm{C}$ and relaxation for $5 \mathrm{~min}$. The $\mathrm{Si}$ wafer was then mounted onto the chamber of a photolithographic machine. The wafer was exposed to UV $\left(\lambda=365 \mathrm{~nm}\right.$, dose $\left.=150 \mathrm{~mJ} \mathrm{~cm}^{-2}\right)$ for $150 \mathrm{~s}$ with a photomask having a microhole array, followed by $5 \mathrm{~min}$ of relaxation. After the UV exposure, the wafer was further baked at $100{ }^{\circ} \mathrm{C}$ for $3 \mathrm{~min}$ and relaxed for $5 \mathrm{~min}$. Subsequently, the UV-exposed SU-8 layer was developed using an SU-8 developer (Microchem Corp, Westborough, MA, USA) for $5 \mathrm{~min}$, followed by rinsing with isopropyl alcohol and blow-drying with nitrogen. The SU-8 film was hard-baked again at $150{ }^{\circ} \mathrm{C}$ for $10 \mathrm{~min}$ to produce a SU-8 template. Finally, the substrate was rinsed with deionized water and blow-dried with nitrogen. For surface hydrophobization, trichloro $(1 \mathrm{H}, 1 \mathrm{H}, 2 \mathrm{H}, 2 \mathrm{H}$-perfluorooctyl) silane $\left(\mathrm{CF}_{3}\left(\mathrm{CF}_{2}\right)_{5} \mathrm{CH}_{2} \mathrm{CH}_{2} \mathrm{SiCl}_{3}\right)$ was spray-coated onto the $\mathrm{SU}-8$ template surface.

\subsection{Fabrication of PDMS Micropillar Array}

The PDMS micropillar array was prepared by employing the SU-8 template. In total, $400 \mathrm{mg}$ of a mixture (wt ratio 9:1) of the prepolymer (Sylgard 184A) and curing agent (Sylgard 184B) was spin-coated onto the SU-8 template at a speed of $1000 \mathrm{rpm}$ for $5 \mathrm{~min}$ and then transferred into a vacuum chamber for degassing $(10 \mathrm{~min})$. Subsequently, it was placed in a convection oven and cured at $70{ }^{\circ} \mathrm{C}$ for $2 \mathrm{~h}$, resulting in a high-modulus PDMS micropillar film. A second layer of PDMS mixture (10:1 mixture of Sylgard 184A and Sylgard 184B) was spin-coated onto the first film which then underwent the same degassing and curing processes. Finally, the fused PDMS micropillar array film was carefully peeled off from the SU-8 template.

\subsection{Fabrication of the GnPs/AgNWs Strain Sensor Component}

A polyvinylidene fluoride (PVDF) film template with the desired design was fabricated via a laser cutter with a preset program to produce a desired shape and sandwiched over a nylon filter membrane in a vacuum filtration setup. Afterwards, $12 \mathrm{~mL}$ of a graphene nanoplatelets/acetone dispersion at a concentration of $\sim 0.5 \mathrm{mg} / \mathrm{mL}$ and $10 \mathrm{~mL}$ of an AgNW aqueous dispersion at $\sim 0.1 \mathrm{mg} / \mathrm{mL}$ were alternately filtered through a nylon filter membrane, resulting in a sandwich structure of the hybrid materials. The graphene nanoplatelets are hydrophobic and cannot uniformly disperse in an AgNW aqueous dispersion, so alternative filtration can easily produce the sandwich structure. The filtration of either the graphene nanoplatelets/acetone and AgNW aqueous dispersion continued for $1 \mathrm{~min}$. To increase the weight percentage of AgNW in the composite, a higher amount of AgNW aqueous solution was employed in the filtration process accordingly.

After filtration and drying, a small amount of freshly mixed PDMS $(\sim 0.1 \mathrm{~g})$ was poured and permeated into the GnPs/AgNWs composite. The PVDF film template was then removed, and the nylon filter membrane, bearing the PDMS-wetted GnPs/AgNWs composite, was placed face down onto the PDMS micropillar array. After curing of the PDMS-wetted GnPs/AgNWs composite onto the PDMS micropillar array, Ag wires were bonded onto the two terminals of the patterned GnPs/AgNWs composite via an Ag paste. More details of the process are shown in Supplementary Materials. 


\subsection{Fabrication of the Drug Delivery System}

A chitosan hydrogel $(1 \mathrm{wt} \%)$ was prepared in acetic acid solution $(1 \% v / v)$ and incubated in a water bath for $3 \mathrm{~h}$ at $55^{\circ} \mathrm{C}$. Specific nanoparticles were prepared using the standard double-emulsion method. Briefly, polylactic-co-glycolic acid with fluorescent AlexaFluor (AF488) tag (PolySciTech, West Lafayette, IN, USA) was dissolved in methylene chloride. After introducing the model drug deferoxamine in distilled water, the mixture was emulsified by sonication and then sonicated again with $2 \%$ polyvinyl alcohol. The prepared double emulsion was then purified by vacuum evaporation and centrifugation. The deferoxamine-polylactic-co-glycolic acid nanoparticles (deferoxamine-PLGANPs) were then mixed vigorously overnight to form composite hydrogel scaffolds. The scaffolds were kept at $4{ }^{\circ} \mathrm{C}$ in the dark before being applied to the strain sensors. The permeability of the nanoparticles was measured by the fluorescent signal of the fluorescent agent (AF488)-labelled PLGANPs in chambers.

\subsection{Coating the Drug Delivery System onto the Surface of the Wearable Strain Sensor}

The strain sensor surface was first treated with oxygen plasma in a chamber at $500 \mathrm{mTorr}$ pressure. The plasma was produced at $50 \mathrm{~W}$ with an oxygen flow rate of $20 \mathrm{sccm}$. The exposure time was $20 \mathrm{~s}$. Then, $\sim 5 \mathrm{~g}$ chitosan hydrogel was drop-casted on the sensor surface over an area of $\sim 16 \mathrm{~cm}^{2}$. The sensor was then placed in a vacuum chamber at $40{ }^{\circ} \mathrm{C}$ for $20 \mathrm{~min}$ to remove excess water, producing a thin film on the sensor.

\subsection{Preparation of a Cellular Migration Transwell Model}

Human epidermal keratinocytes HaCaT cells and human skin fibroblasts HS27 cells were cultured in Dulbecco's modified Eagle's medium supplemented with $10 \%$ fetal bovine serum and $1 \%$ penicillin/streptomycin solution at $37^{\circ} \mathrm{C}$ in a cell culture incubator. The cells were passaged twice per week using trypsin before culturing on commercially available Transwell ${ }^{\circledR}$ systems (Corning, Lowell, MA, USA). This six-well plate-based system contains semipermeable polycarbonate membrane inserts which separate the apical and basolateral compartments. To form in vitro physiological adult skins, we used a co-culture system. The keratinocytes were seeded on top of the Transwell ${ }^{\circledR}$ membrane to form the outer barrier layer representing the epidermis, while the skin fibroblasts were seeded on the other side of the Transwell@membrane to form the underlying dermis. After two weeks of cell culture, the cellular skin model was ready to be used for further study. The preparation of such a system is detailed in Section 14 of the Supplementary Materials.

\subsection{In Vitro Drug Penetration through the Cellular Transwell Model}

PLGANPs-loaded chitosan hydrogel scaffolds were applied to the surface of the strain sensors. The sensors were dried under vacuum to make sure all the solvents were evaporated completely before the drug penetration test. The scaffolds were added onto the in vitro skin model and incubated for 6 or $12 \mathrm{~h}$. After incubation, the cells on the Transwell membrane were fixed with $4 \%$ paraformaldehyde, permablised with $1 \%$ Triton X, stained with 4',6-diamidino-s-phenylindole and embedded in ProLong Gold mounting medium. The drug-loaded nanoparticle penetration was imaged using the Z-stack function of the confocal microscope.

\subsection{Ex Vivo Skin Permeation of Polylactic-co-glycolic Acid Nanoparticle}

The ex-vivo penetration study was performed using intact porcine skin following the methods previously described $[28,29]$. Intact full-thickness skin from white pigs $(n=2)$ was collected from the South Australian Health and Medical Research Institute (Gilles Plains, Adelaide, Australia). After cleaning with $70 \%$ ethanol, the skin was cut into $1 \times 1 \mathrm{~cm}^{2}$ and immediately mounted on a sterile disk, taking care to preserve the natural tension of the skin. The explants were cultured in Dulbecco's modified eagle medium supplemented with $10 \%$ fetal calf serum, $5 \%$ fungizone, and $2 \%$ penicillin/streptomycin at the air-liquid interface, with the epidermis being exposed to air, and the 
dermis being submerged in the medium. Approximately $200 \mu \mathrm{L}$ of chitosan hydrogel containing PLGANP, loaded with deferoxamine and labeled by Alexa-Flour 488, was applied on the surface of porcine skin $(n=6)$. The explants were returned to the $37^{\circ} \mathrm{C}, 5 \% \mathrm{CO}_{2}$ incubator and incubated for $6 \mathrm{~h}$ and $12 \mathrm{~h}$. The explants were then sectioned, fixed in $10 \%$ formalin overnight and subjected to routine histological tissue processing [30]. Paraffin-embedded formalin-fixed sections were analyzed using a routine immunohistochemistry protocol described previously [28].

Sections of the skin were processed for immunohistochemical detection of Alexa Flour 488-labelled deferoxamine-loaded PLGANPs. Briefly, slides were washed with phosphate buffered saline and incubated with $4^{\prime}, 6$-diamidino-s-phenylindole (1:5000) for $3 \mathrm{~min}$. Following the washing, the slides were treated with Dako fluorescent mounting medium. The slides were stored in the dark at $-20^{\circ} \mathrm{C}$ until imaging, using an Olympus fluorescent microscope (Olympus, Shinjuku-ku, Tokyo, Japan). Image analysis was performed using the CellSens program. The localization of PLGANPs and depth of penetration in the epidermis ere determined using CellSens program.

\subsection{Device Characterization}

SEM micrographs were obtained using a FEI Helios Nanolab 600 ion beam microscope (Thermo Fisher Scientific, Hillsboro, OR, USA) (operating voltage $2 \mathrm{kV}$ and current $60 \mathrm{pA}$ ). Transmission electron microscopy (TEM) images were made using a Philips CM200 at $200 \mathrm{kV}$. Samples for TEM were prepared via dispersion in tetrahydrofuran $(0.0004 \mathrm{wt} \%)$ through $30 \mathrm{~min}$ sonication and drop-casted on 200-mesh copper grids, followed by drying in air. Bright-field high-magnification TEM images were taken with a JEOL 2100F microscope (JEOL, Akishima, Tokyo, Japan) operated at $120 \mathrm{kV}$. Raman spectra were recorded by a Renishaw in Via Raman microspectrometer with $633 \mathrm{~nm}$ laser excitation and notchfilter cutting at $\sim 100 \mathrm{~cm}^{-1}$. Measurements were carried out at 4.00 to $0.04 \mathrm{~mW}$ incident power to avoid sample damage or laser-induced heating. The changes of resistance were recorded by an electrochemical workstation CHI660E chronoamperometric technique. For strain sensor testing, the working voltage was set to 0.5 V. For higher sensitivity, a digital source meter Keithley 2602 was employed to collect the electrical response of the strain sensors. The tensile strain characterization of the sensor was performed with an Instron system 5567, with simultaneous measurement of resistivity characteristics recorded with the source meter Keithley 2602 or with the electrochemical workstation CHI660E. The sensor durability was investigated by applying cyclic loading at a frequency of $5 \mathrm{~s}$ per cycle, using an Instron system 5567; all sensors were stretched at strain values of $5 \%$ and $10 \%$. The pressure-sensing performance of the sensor was recorded by placing poise weights with weight from $1 \mathrm{~g}$ to $100 \mathrm{~g}$ or small pieces of silicon with weight of $20 \mathrm{mg}$ onto the sensor and recording the resistivity. During the pressure-sense testing, the sensor was placed onto the bench top. A Zeiss 780 NLO confocal laser scanning microscope (Zeiss, Oberkochen, Germany) was used to observe the penetration of PLGANP in the epidermis of porcine skin.

\section{Results}

\subsection{Fabrication of the SU-8 Template and PDMS Substrate}

The overall concept of our multifunctional strain sensor is depicted in Figure 1. It is made up of three key components: a dry adhesive substrate (PDMS micropillars array), a patterned strain-sensing composite component ( $\mathrm{GnPs}$ and $\mathrm{AgNWs}$ ) and a transdermal drug delivery system (drug-vessel-embedded chitosan hydrogel).

The fabrication began with the preparation of micro-holes in an SU-8 photoresist template on a 5 inch silicon wafer. Then, high-modulus PDMS precursors were spin-coated on the template to mold PDMS micropillars, after coating the low-modulus PDMS precursor. The whole fabrication procedure is schematically shown in Figure S1. When the high-modulus PDMS micropillars (2.81 MPa) attach on skin, they can form a robust, adhesive interface with the skin surface, and the low-modulus PDMS film will became a soft, flexible substrate. 
Figure S2a,c show top-down images of the SU-8 template that has microholes which could be filled with PDMS. The PDMS film (Figure S2b) had a uniformly patterned array of micropillars of $\sim 10 \mu \mathrm{m}$ in dimeter, as shown in Figures S2d and S3a. The cross section of these micropillars in Figure S3b indicates that these micropillars had an aspect ratio of $\sim 3: 1$. A micropillar film is advantageous over a flat film in terms of adhesion, and the mechanism is illustrated in Figure S2f,g. When a flat adhesive film contacts a rough surface, contact is only made at the highest asperities, and the deformation of the bulk layer is relatively small (Figure S2f). In contrast, each micropillar can deform to maintain a large contact area with the target surface (Figure S2g). Many micropillars deform to create sufficient contact area and thus they have higher adhesion performance than a completely flat PDMS film [31]. Figure S3c shows the attachment of micropillars over a relative rough surface.

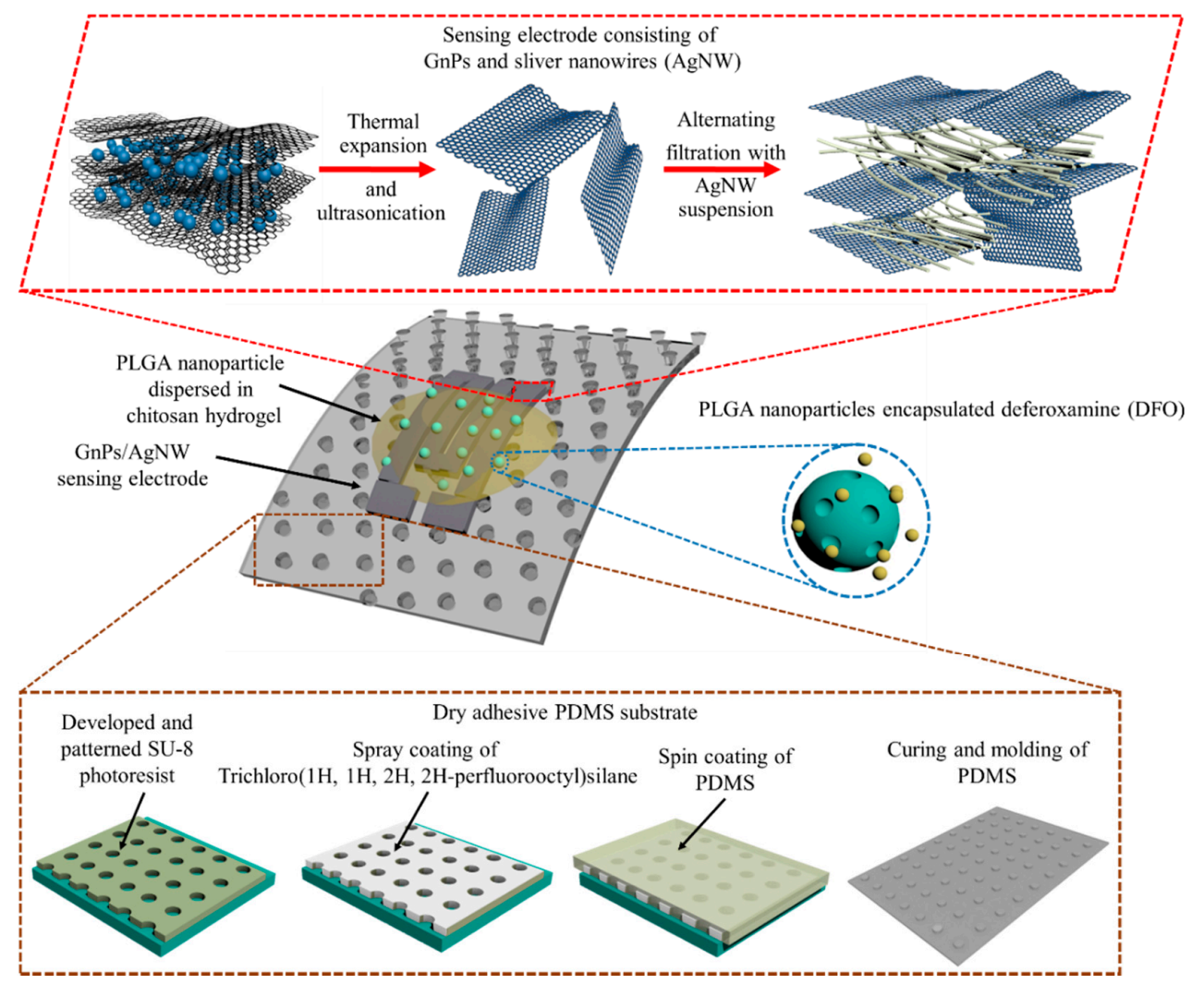

Figure 1. Schematic illustration of the multifunctional sensor with a flexible strain-sensing component and a transdermal drug delivery system. GnPs, graphene nanoplatelets, AgNWs, silver nanowires, PLGA, polylactic-co-glycolic acid, PDMS, polydimethylsiloxane.

The micropillar film had a pull-off strength of $\sim 1.0 \mathrm{~N} / \mathrm{cm}^{2}$, as indicated by its ability to hold a large alloy block $(\sim 0.5 \mathrm{Kg})$ (Figure S2e). It could easily adhere onto a silicon wafer (Figure S2h) or human arm for over $5 \mathrm{~h}$ (Figure S2i). A small piece of the film $\left(1 \times 1 \times 0.1 \mathrm{~cm}^{3}\right)$ attached to a glass sheet could sustain a load of $100 \mathrm{~g}$ for over $2 \mathrm{~h}$ (Figure S4). Therefore, the PDMS micropillar film would be a feasible substrate for the development of skin-mountable electronic devices. After fabrication, the exposed PDMS area of each sensor would be $\sim 5 \mathrm{~cm}^{2}$, thus it could provide enough strength to maintain the attachment of a sensor on skin.

\subsection{Strain Sensor Fabrication and Properties}

We employed a "filtration-patterning" method to fabricate the sensor composite consisting of GnPs and AgNWs, which are respectively characterized in Sections 5 and 6 of the Supplementary Materials. Using vacuum filtration, $\mathrm{GnP}$ and $\mathrm{AgNWs}$ were alternately deposited on filter paper, creating a sandwich-structured $\mathrm{GnP} / \mathrm{Ag}$ film whose geometry was similar to the pattern on the 
PVDF film (Figures S7 and 2a). More detail of the fabrication process is indicated in Section 8 of the Supplementary Materials. To improve the durability, $100 \mathrm{mg}$ of PDMS was spin-coated onto the sensor, followed by curing; in this process, PDMS would diffuse into the GnP/Ag sandwich film. The cross section area of a GnP/Ag sandwich film encapsulated by PDMS is shown in Figure S9. In the $\mathrm{GnP} / \mathrm{Ag}$ sandwich composite $\sim 40 \mu \mathrm{m}$ in thickness, most GnPs would either overlap or interleave each other, thus coming into extensive contact (Figure 2b). Likewise, AgNWs would interconnect to form a network (Figure 2c). With stretching, GnPs would slide past each other and separate, exhibiting piezoresistivity; AgNWs would maintain a conducting network to accommodate the load.
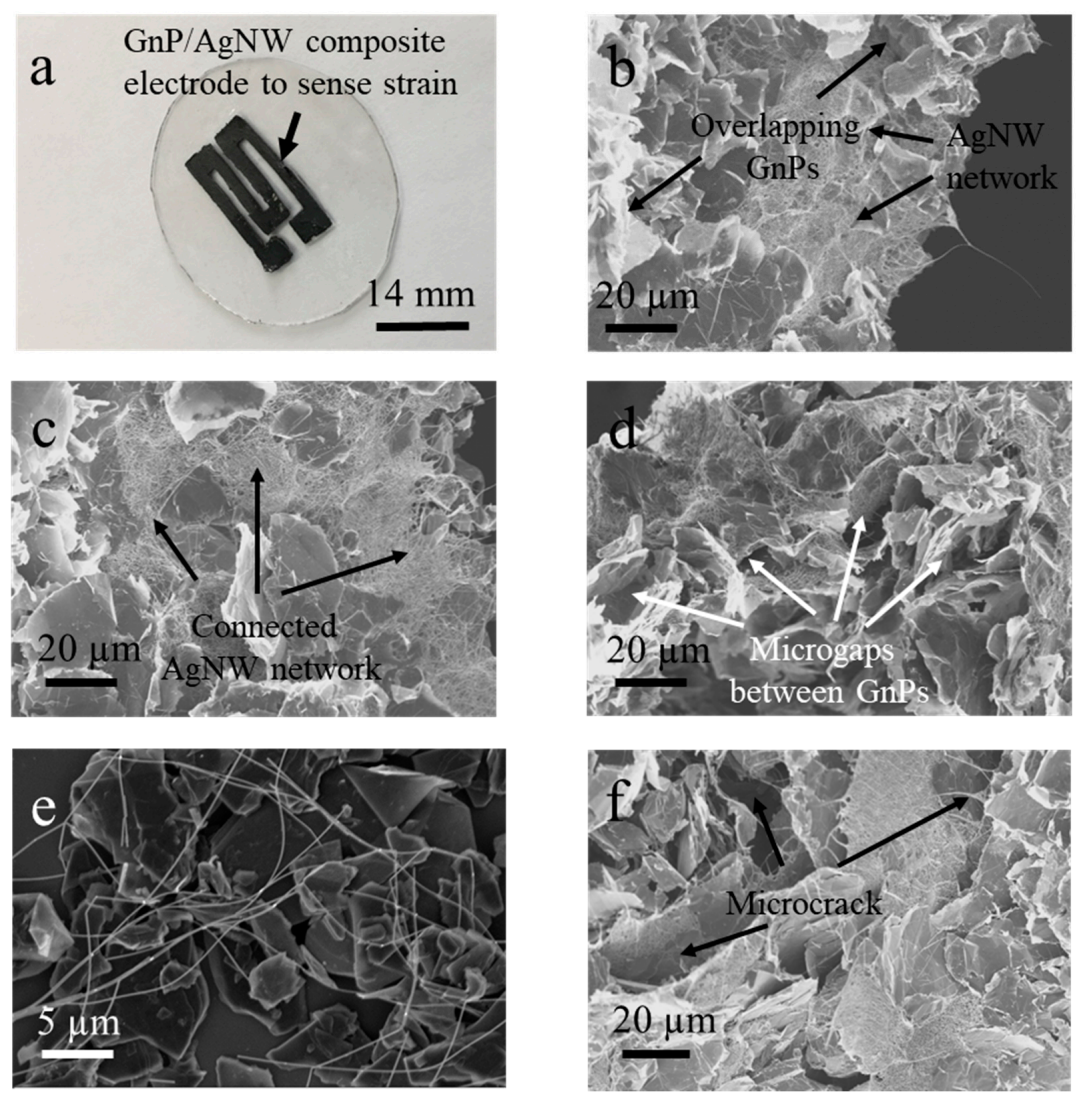

Figure 2. Digital photographs of (a) two sensor components were transferred onto a PDMS substrate and SEM micrographs of (b) GnP/AgNW composite, (c) cross section of the composite without strain, (d,e) GnPs with micro-gaps and connected by AgNWs at a strain of $~ 5 \%$, (f) microcracks formed by separated GnPs and AgNWs at a strain of $\sim 10 \%$. Characterization of flexible $\mathrm{GnP} / \mathrm{Ag}$ strain sensors.

The sensitivity of the pizeoresistivity was evaluated by a gauge factor (GF) [32], calculated as

$$
G F=\left(\Delta R / R_{0}\right) / \varepsilon
$$

where $\Delta R$ is the resistance change of the sensor under strain, $R_{0}$ is the resistance prior to straining, and $\varepsilon$ is the applied strain. The piezoresistivity can be mainly attributed to three mechanisms: (i) tunneling resistance change between adjacent nanoplatelets caused by microcracks, (ii) the influence of geometrical changes of the sensor components, and (iii) the piezoresitivity of individual nanoplatelets due to their deformation [33-35]. The resistance change $\left(\Delta R / R_{0}\right)$ can be expressed as:

$$
\Delta R / R^{0}=(1+2 v) \varepsilon+\Delta \rho / \rho_{0}
$$

where $v$ is Poisson's ratio, $\Delta \rho$ is the resistivity variation, $(1+2 v) \varepsilon$ refers to the influence of geometrical changes, and $\Delta \rho / \rho_{0}$ indicates the piezoresistivity. 
Under tensile loading, GnPs would initially deform and then slide pass each other to form tunneling junctions with a distance of $\sim 3 \mathrm{~nm}$. With further loading, the tunneling width would extend into some micro-gaps between GnPs (Figure 2d). A high-resolution SEM micrograph shows such micro-sized gaps (Figure 2e). According to the transfer length method, the tunneling resistance $\left(R_{t}\right)$ can be evaluated by [36]:

$$
R t=\rho_{s h}(d / W)+2 \rho_{c} / L W
$$

where $\rho_{s h}$ is the sheet resistance of individual nanoplatelets in the channel, $d$ is the tunnel length, $W$ is the tunnel width, and $\rho_{c}$ is the contact resistance between the nanoplatelets. As the width of the tunneling junctions is increased, the tunneling resistance will increase as a result of the increased contact resistance. Then, under larger strain, some GnPs will lose electrical contact with each other, as a result of the deformation of the PDMS, leading to microcracks (Figure 2f). The width of a microcrack (w) can be expressed as:

$$
w=w_{0}(1+C \varepsilon)
$$

where $\varepsilon$ is the tensile strain, and $w_{0}$ is the initial width of a microcrack.

Because of the expansion of microcracks, the contact resistance between GnPs continuously increased. Under a sufficiently large strain, the microcrack expanded, and the some of the GnPs were disconnected. Consequently, the GnPs network provided high piezoresistivity to the composite, while AgNWs changed wave amplitudes and slid across each other to maintain the electrical conductivity.

As a result of the high stretchability of the AgNWs network (up to $40 \%$ in tensile strain $[23,37]$ ) and the high piezoresistive sensitivity of stacked GnPs, a GnP/AgNW composite sensor would have both stretchability and sensitivity. The relative resistance changes of sensors consisting of single GnPs and AgNWs as a function of tensile strain are indicated in Section 10 of the Supplementary Materials. Compared with the sinlge GnPs or AgNWs, the combined materials can provide more linear response of resistivity to strain. Combined with the advantage of a dry adhesive substrate, our sensor can durably attach onto the human body to detect human motion (Figure 3a). The PDMS micropillars would mount on the skin to provide strong adhesion, and the GnP/AgNW composite that is the black region in the sensor would attach on the skin as an electrode to exhibit strong piezoresistivity. On the basis of the theoretical Equation (4), the average gauge factor of the piezoresistive sensor could be estimated as $\sim 33$. As a result of the effect of complicate geometrical factors, the experimental curves were less linear than the theoretical curves.

To investigate the effect of AgNW networks inside the sensor composite, we fabricated sensors with different weight ratios of AgNWs to GnPs: 1:3, 1:6 and 1:9. Figure $3 b$ compares the time-dependent relative resistance of the three sensors. All three sensors could maintain a relatively linear resistance response to strains of up to $22 \%$. At the AgNW/GnP ratio of 1:9, the sensor showed the largest gauge factor of 65.1 with less linear response of resistance change up to a strain of $22 \%$. Increasing the AgNW content led to decreased sensitivity with a more linear response. For example, the sensor with a ratio of 1:3 (AgNW/GnP) showsd the most linear response and lowest gauge factor of 16.2. To achieve a balance between having a linear response and a high gauge factor, we chose a ratio of 1:6 (AgNW/GnP), corresponding to a gauge factor of 41.5 for the following studies.

The above results illustrate that the AgNW network was able to maintain the composite conductivity under large loading; however, it could result in lower sensitivity. In contrast, the GnP stacked structure would lead to a high gauge factor, but, as microcracks expanded, the resistance would increase sharply, resulting in a nonlinear response. The sensor composite can combine both advantages. Under a strain less than $15 \%$, GnPs would stretch and slide past each other, which likely leads to microcracks and a piezoresistive response [38]. Meanwhile, the AgNW network would deform to accommodate the strain and thus serve as an electron-transferring bridge [39]. As a result of the bridge effect of the AgNWs, the resistance can increase steadily rather than sharply, resulting in a relatively linear resistance response to loading. 
a
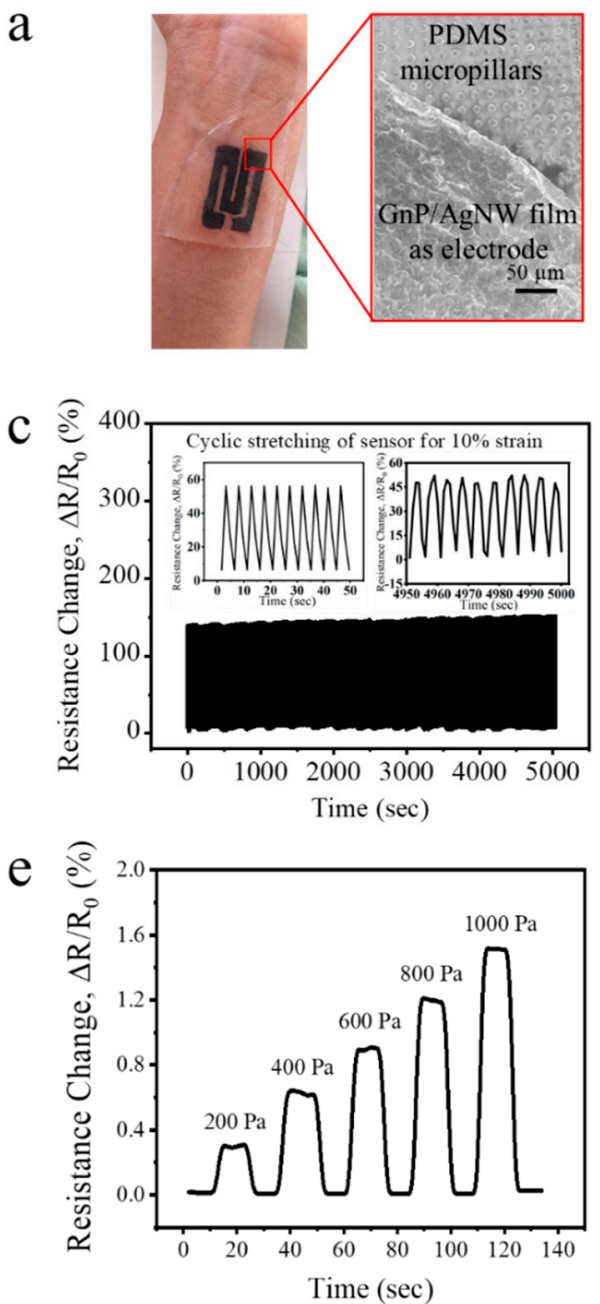
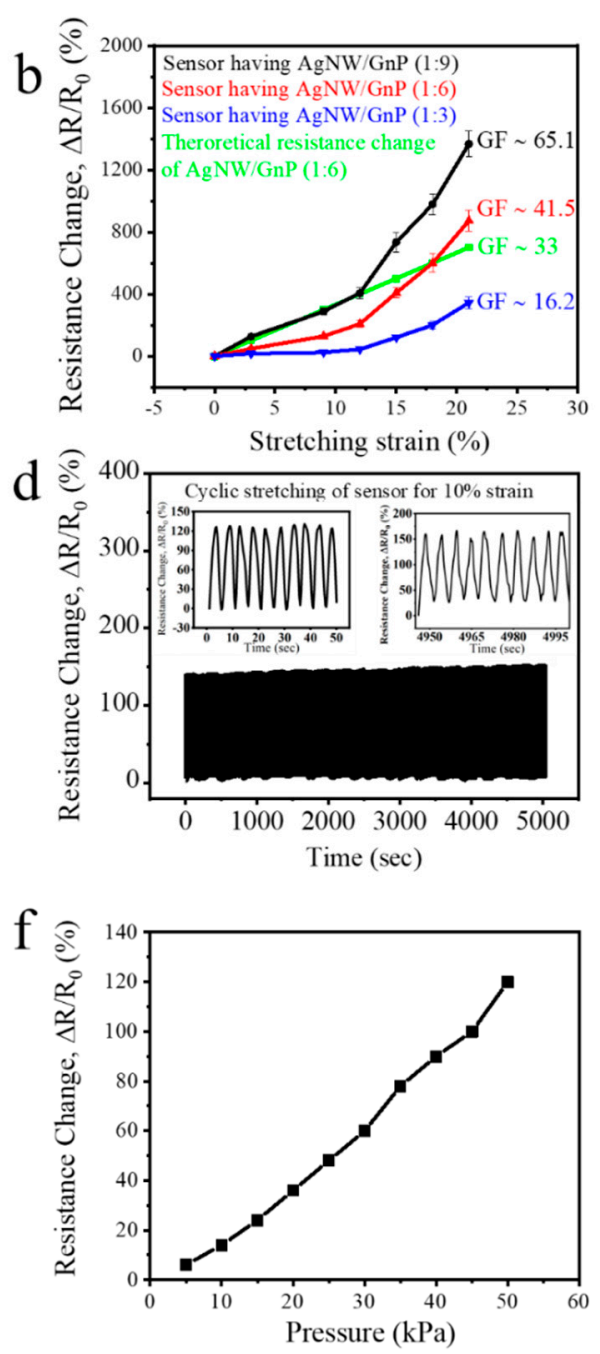

Figure 3. (a) Photographs of a strain sensor attached onto the wrist, with adhesion as strong as that of an adhesive tape. The insets are SEM images for top view of the sensor. (b) Strain-dependent relative resistance change $\left(\Delta R / R_{0}\right)$ and gauge factors of sensors with different weight percentages of AgNWs, (c,d) resistance change $\left(\Delta R / R_{0}\right)$ over 1000 cycles for strains at a frequency of $0.2 \mathrm{~Hz}$ (five seconds per cycle); insets show the enlarged view of the records in the initial (left) and last 10 cycles (right). (e) Real-time resistance changes of the strain sensor with AgNW/GnP ratio of 1:6 at different pressures of 200-1000 $\mathrm{Pa}$ and (f) relative resistance changes of the strain sensor versus the applied pressure ranging from 5 to $50 \mathrm{kPa}$.

The sensor was encapsulated with PDMS, so it exhibits high durability, as shown in Figure 3c,d. It responded almost instantaneously to cyclic loading with remarkable stability and reproducibility even after 1000 cycles at $5 \%$ and $10 \%$ strain at a frequency of $0.2 \mathrm{~Hz}$; as shown in Figure 3c,d inset, the resistance over the last 10 cycles still responded immediately to the strain. Comparing the initial and final 10 cycles, we found that the sensor showed a consistent response to load.

In addition, pressure loading was applied onto the sensor. Pressure sensitivity $(S)$ is defined as [40]:

$$
S=\left(\Delta R / R_{0}\right) / \Delta P
$$

where $\Delta R$ is the resistance change of the sensor under strain, $R_{0}$ is the resistance prior to straining, and $\Delta P$ is the applied pressure.

Throughout a step-by-step increase of pressure from 200 to $1000 \mathrm{~Pa}$, the sensor presented a fast and linear increase in resistance and a pressure sensitivity of $\sim 1.5 \mathrm{kPa}^{-1}$ (Figure 3e). This pressure 
sensitivity can be explained by the expansion of tunneling and micro-gaps between GnPs under low pressure. At large pressures ranging from 5 to $50 \mathrm{kPa}$, which correspond to the pressure from a pule and a powerful thumb press, the sensor exhibited a slightly higher pressure sensitivity of $\sim 1.99 \mathrm{kPa}^{-1}$ (Figure 3f). The slight increase in pressure sensitivity can be attributed to an increase in the width of the microcracks between GnPs.

By using custom-made software to control the activation time of an Instron machine and a CHI electrochemical work station, the sensor response time was measured to be less than $50 \mathrm{~ms}$, which is comparable to the response time of human skin to external stimuli (30-50 ms) [41,42]; relevant details are shown in Section 11 of the Supplementary Materials. In addition, the properties of the GnP/ AgNW stain sensor were compared with those of other strain sensor in Section 12 of the Supplementary Materials. Compared with previous work [43], our strain sensor has the capability to measure pulse motion and high durability for 1000 cycles.

\subsection{Application of the Strain Sensor as Health Monitor, Electronic Skin}

Featuring wearability, sensitivity and reliability, our wearable sensors can be adopted for real-time monitoring of various human motion signals. Firstly, the sensor can detect strain associated with wrist bending (Figure 4a) and finger touching (Figure 4b). During wrist bending, two peaks can be recorded. The first peak could be caused by the movement of extensor indicis toward the wrist [44], and the second peak could be attributed to the bulge movement of wrist skin. When such forms of strain are applied to the sensor, the composite film either stretches or compresses, resulting in an obvious resistance increase. Once the strain is removed, the film returns to its original geometry and position.
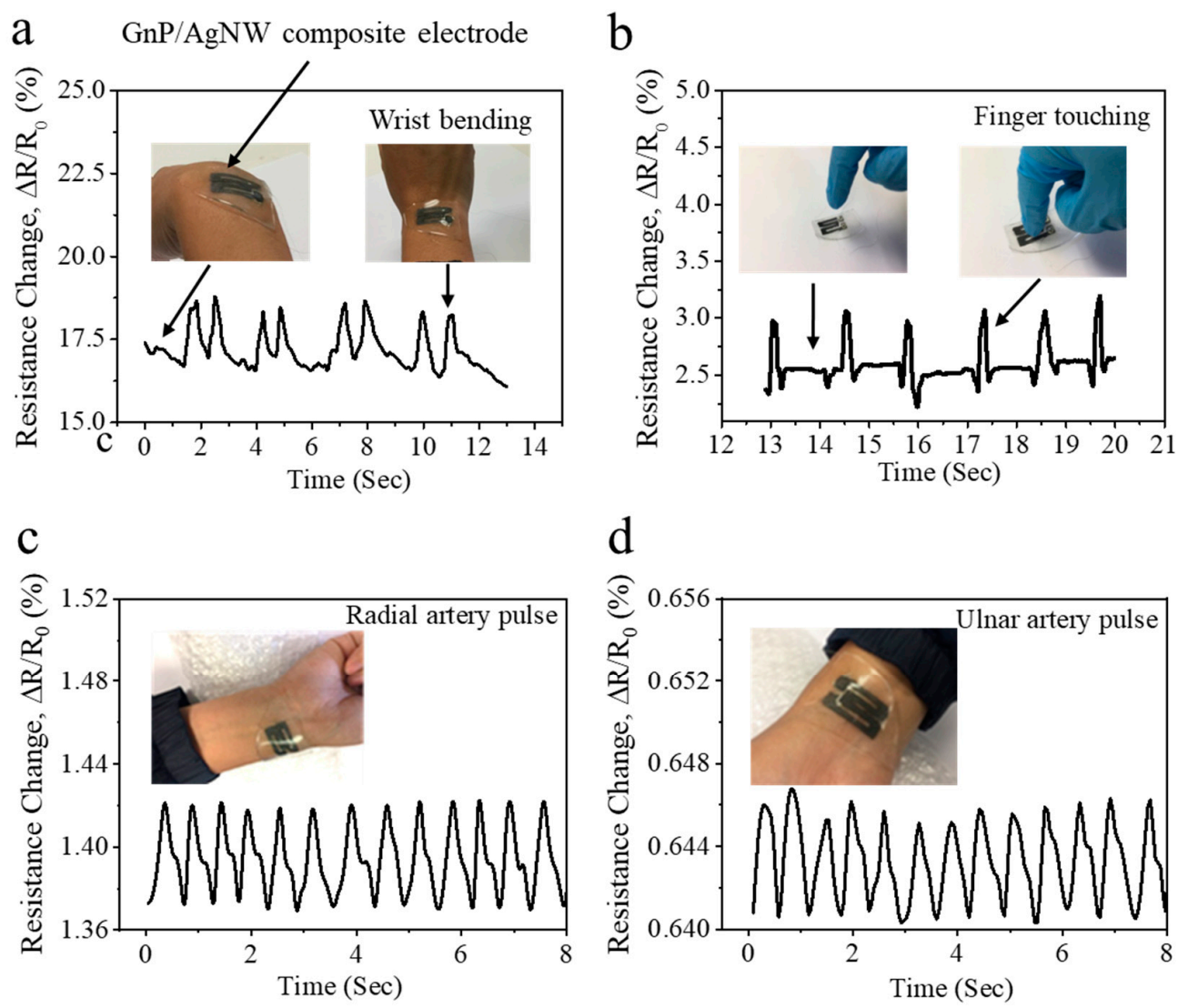

Figure 4. Applications of strain sensors. Real-time monitoring of human motion by detecting resistance change during (a) wrist bending, (b) finger touching, (c) radial artery pulse and (d) ulnar artery pulse. The insets are the digital photos of a sensor attached on skin or put on a table for testing. The black region of the sensor is the $\mathrm{GnP} / \mathrm{AgNW}$ composite electrode that produces piezoresistivity. 
Secondly, as a result of the use of a dry adhesive substrate for creating robust, conformal contact between sensor and skin, even a slight strain from a pulse at $~ 1.5$ beat per second could be clearly detected by the sensor (Figure 4c). Each pulse cycle recorded at the wrist included (i) a peak corresponding to the precussion wave, (ii) a relative steady section originating from the diastolic wave, and (iii) a minor dicrotic notch [45]. The pulse measurement could also be made at other body locations, including the radial and ulnar arteries (see Figure S12 for anatomical locations of radial and ulnar arteries; testing graphs are shown in Figure $4 \mathrm{c}, \mathrm{d}$ ). The pulse signals of the radial artery and ulnar artery exhibited almost the same of $\sim 90$ beat per min, but the radial pulse had higher beating intensity than ulnar artery pulse.

Through examining an enlarged pulse signal, as shown in the Section 13 of the Supplementary Materials, the heights of the precussion wave, tidal wave and dicrotic wave could be identified [46,47]. By calculating the heights and time interval between precussion wave and dicrotic wave, we obtained the augmentation index $\sim 25.4 \%$ and reflection index $\sim 5.5 \mathrm{~ms}^{-1}$. This result indicates the potential of using the sensor for diagnostic monitoring.

Electronic skin (E-skin) has attracted growing attention due to its unique capability to detect subtle pressure changes for wearable health monitoring, sensitive tactile information acquisition, invasive surgery and prosthetics [48]. The sensor herein could be also used as a stretchable E-skin to instantaneously detect the slight motion of a field cricket and a wild snail (Figure S14).

\subsection{In Vitro and Ex Vivo Delivery Capability of the Transdermal Drug Delivery System}

As illustrated in Figure S2i, the dry adhesive substrate has the capability to form robust, stable interaction with human skin. A promising possibility is to create a multifunctional device that can simultaneously administer drugs and record physiological responses. To demonstrate this possibility, our strain sensor was modified to allow for transdermal delivery of drugs.

A PLGANP system where a model drug, deferoxamine (DFO), was embedded inside each nanoparticle was used as a drug delivery vehicle. The PLGANP vehicles were dispersed in a chitosan hydrogel. The preparation of this system is detailed in the Section 16 of the Supplementary Materials. The drug delivery hydrogel system was coated onto the wearable PDMS patch (on the side facing the skin) by drop-casting, to create an integrated sensor. The entire device was placed in a vacuum chamber at $40^{\circ} \mathrm{C}$ for $20 \mathrm{~min}$ to remove excess water and dry the thin film (Figure S17). Subsequently, the sensor was cut into pieces of $1 \times 1 \mathrm{~cm}^{2}$ and UV-sterilized for the drug delivery tests.

We first built an in vitro cell model which mimicked the structure of human skin to observe the penetration process of the embedded nanoparticles. As shown in Figure S18, the cell model contained a cell transwell chamber, a polycarbonate porous membrane and two cell layers of keratinocytes and skin fibroblasts. After incubation for 6 and $12 \mathrm{~h}$, the transwell membranes with the cell layers were collected and imaged using a confocal microscope.

Confocal micrographs indicated that: (i) the cell location was defined by a cellular nuclei marker 4',6-diamidino-s-phenylindole (DAPI) molecules (Figure 5a); (ii) the two cellular layers mimicking epidermis and dermis were uniformly grown on the top and bottom surfaces of the membrane prior to the drug penetration test (Figure 5b); (iii) after 6 and $12 \mathrm{~h}$, more nanoparticles had penetrated through the epidermis cell layer and dispersed inside the dermis cells (Figure $5 \mathrm{c}$ ). The result shows that the nanoparticles had a penetration depth of $19.03 \pm 2.15 \mu \mathrm{m}$ after $6 \mathrm{~h}$ of incubation, and the depth increased to $31.90 \pm 2.46 \mu \mathrm{m}$ after $12 \mathrm{~h}$ (Figure $5 \mathrm{~d}$ ). This in vitro penetration study suggested that the drug DFO-PLGANPs could efficiently penetrate into the dermis.

To further investigate the penetration of PLGANPs into skin, we applied the system onto intact porcine skin to demonstrate permeability. Porcine skin has a physiological structure very similar to that of human skin in terms of thickness of the epidermis and dermal-epidermal thickness ratio [24,37,49]. A typical photo of the testing rig is shown in Figure S19, where a number of sensors, each $1 \times 1 \mathrm{~cm}^{2}$, were placed onto the porcine skin piece to mimic the transdermal drug delivery process. 


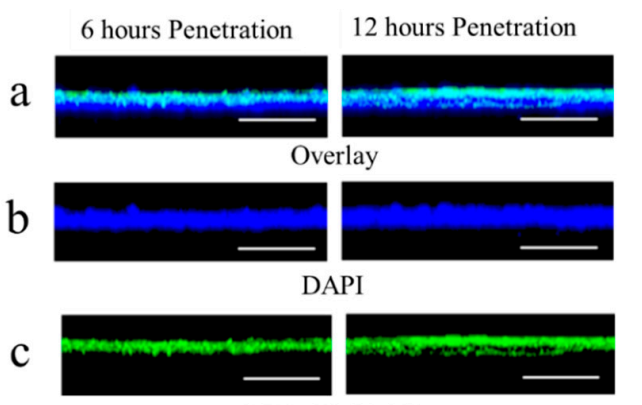

DFO- PLGA NPs

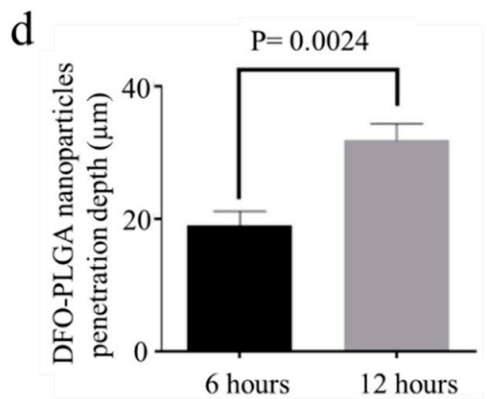

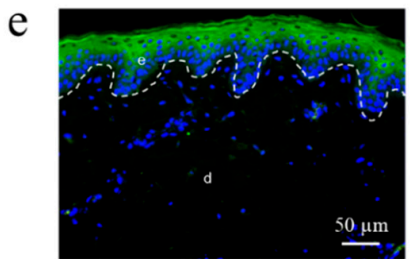

6 hours Penetration

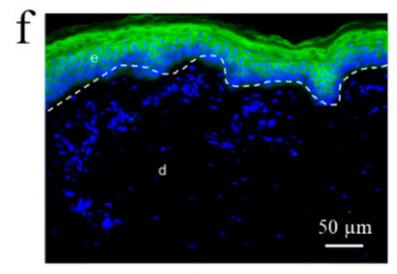

12 hours Penetration

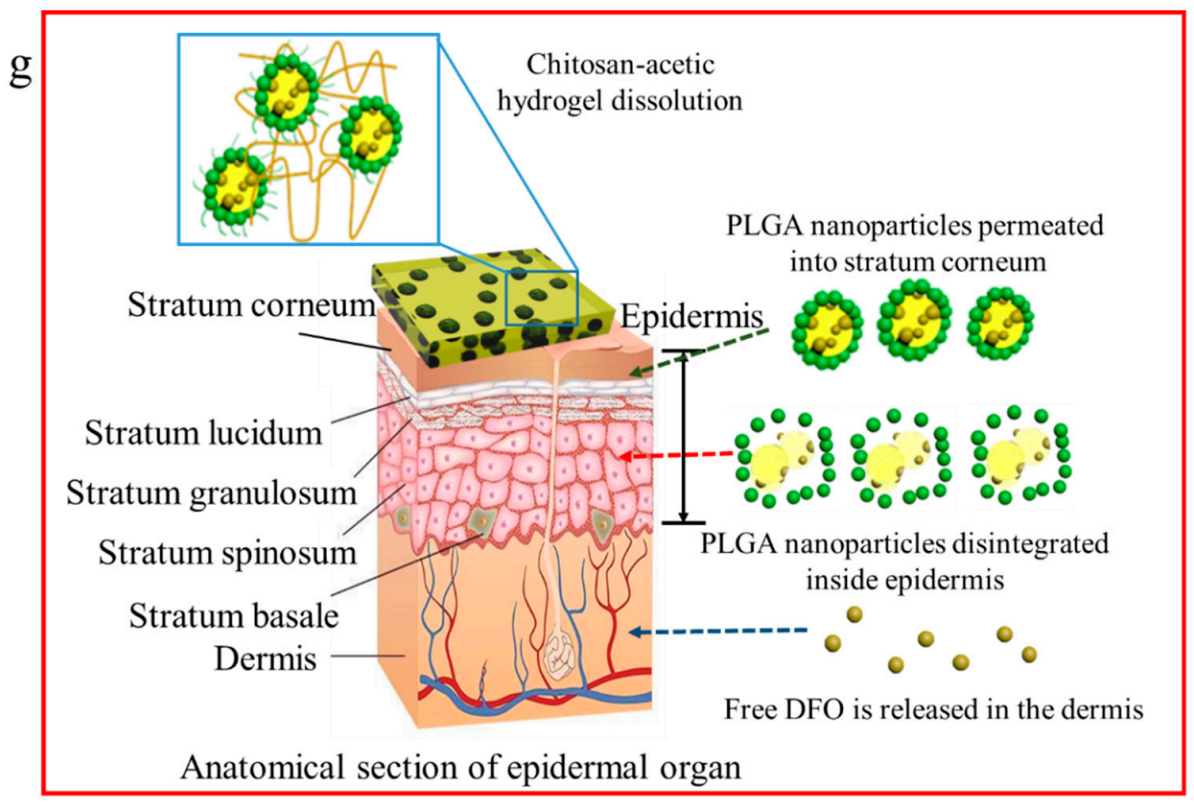

Figure 5. $(\mathbf{a}-\mathbf{c})$ Confocal images of a cross section of the transwell membrane with two cell layers after incubation for 6 and $12 \mathrm{~h}$. (d) Penetration depths of DFO-PLGANP into the cell layers. (e,f) Confocal images of a cross section of skin tissue with DFO-PLGANP after incubation for 6 and $12 \mathrm{~h}$. (g) Schematic overview of the transdermal drug delivery process, including coating chitosan-acetic hydrogel on skin surface, PLGA nanoparticles penetration, disintegration and release of DFO.

In Figure 5e,f, PLGANPs were visualized using fluorescence images to assess the penetration degree. The following phenomena were observed. First, after incubation for $6 \mathrm{~h}$ at room temperature, a layer of green florescence formed in the apical epidermis (Figure 5e), indicating the effective release of PLGANPs from the chitosan hydrogel through the sensor; these nanoparticles permeated into the stratum corneum and into the stratum granulosum. Second, after $12 \mathrm{~h}$ of incubation at room temperature, the intensity of the Alexa Flour- 488 signal was stronger, suggesting more nanoparticles had been delivered (Figure 5f). The maximum penetration depth was observed to be $60 \mu \mathrm{m}$, confirming penetration up to the stratum spinosum and stratum basale in the epidermis. Lastly, while the nanoparticles were present in the epidermis, no effective diffusion was observed in the papillary dermis, which may be explained by the nanoparticle size preventing them from reaching the intercellular spaces of the dermis as well as the intact basement membrane. 
The drug release process is depicted in Figure 5g. Initially, the sticky chitosan hydrogel with a large number of PLGA nanoparticle was attached onto porcine skin. Next, as the aqueous solution permeated the skin, these nanoparticles penetrated into the stratum corneum, which represents the main physical barrier of the skin $[49,50]$. Once penetrated through the stratum corneum, the nanoparticles disintegrated and dissolved in the epidermis, releasing DFO [51]. Lastly, in a physiological system, DFO penetration to the epidermis would allow rapid absorption into the circulation through the capillaries [52,53].

DFO is used to treat acute iron intoxication and chronic iron overload due to transfusion-dependent anemia [54]. Hypoxia-inducible factor-1 alpha (HIF-1 $\alpha$ ) accelerates wound closure, but its function is compromised by a high glucose-induced and reactive oxygen species-mediated modification of its coactivator p300 in diabetes [55]. DFO as an iron chelator increases HIF- $1 \alpha$ transactivation in diabetes by preventing iron-catalyzed reactive oxygen stress. Through this wearable device, DFO can be delivered and released into diabetic ulcers or wounds. Such a drug delivery system has the potential to address diabetic ulcers and wounds as well as dermatological conditions with impaired skin barrier.

\section{Conclusions}

In summary, we have developed a novel multifunctional wearable strain sensor comprised of a skin-mountable dry adhesive substrate, a sensing component and a transdermal drug delivery system. The dry adhesive substrate was fabricated using a simple photolithography technique. This technique employed a micropiller array which effectively increased the contact area to skin. In addition, the high modulus of the micropillars led to a strong pull-off strength from the substrate of $1 \mathrm{~N} \mathrm{~cm}^{-2}$. The sensing part consisted of layered graphene nanoplatelets and silver nanowires that displayed high piezoresisitivity for monitoring real-time motion signals, such as finger bending and radial pulse. Since the layered composite was embedded firmly into the PDMS substrate, the sensing performance was stable and reproducible for 1000 stretching cycles at $10 \%$ strain and frequency of $0.2 \mathrm{~Hz}$. It also displayed a good gauge factor of 41.5. In addition, the skin penetration results of the drug delivery system demonstrated that the polylactic-co-glycolic acid nanoparticles effectively penetrated into the stratum corneum reaching $\sim 60 \mu \mathrm{m}$ in depth after $12 \mathrm{~h}$ of incubation. This research work demonstrates the potential of integrating strain-sensing and diagnostic elements to create all-in-one theranostic devices. There may be two limitations of the devices. First, although the dry adhesive substrate can provide a high pull-off strength, after long-term usage, dust would attach on the substrate and thus reduce the pull-off strength. Second, the transdermal drug delivery system relies on the nanocarrier that only can carry low-molecular-weight drugs. In the future, the pull-off strength of the dry adhesive substrate will be enhanced by using more advanced techniques to fabricate various bioinspired adhesives. Besides, the effect of strain on the drug delivery process would also need to be investigated.

Supplementary Materials: The following are available online at http:/ / www.mdpi.com/2311-5629/5/2/17/s1. Author Contributions: G.S., Z.K. and T.L. conceived and designed the experiments; G.S., J.P., Z.K. and T.L. performed the experiments; G.S., T.L. and Z.K. analyzed the data; A.C. and S.E.L. contributed reagents/materials/analysis tools; G.S., S.E.L. and Y.L.Z. discussed the data and wrote the paper. A.C. and Y.L.Z. supervised the project.

Funding: This work was financially supported by the Australian Research Council (LP160101521 and DP170104157).

Acknowledgments: This work was performed (in part) at the South Australian node of the Australian National Fabrication Facility under the National Collaborative Research Infrastructure Strategy.

Conflicts of Interest: The authors declare no conflict of interest.

\section{References}

1. Gong, S.; Cheng, W. One-Dimensional Nanomaterials for Soft Electronics. Adv. Electron. Mater. 2017, 3, 1600314. [CrossRef] 
2. Liu, Y.-F.; Huang, P.; Li, Y.-Q.; Liu, Q.; Tao, J.-K.; Xiong, D.-J.; Hu, N.; Yan, C.; Wang, H.; Fu, S.-Y. A biomimetic multifunctional electronic hair sensor. J. Mater. Chem. A 2019, 7, 1889-1896. [CrossRef]

3. Li, C.; Zhang, D.; Deng, C.; Wang, P.; Hu, Y.; Bin, Y.; Fan, Z.; Pan, L. High performance strain sensor based on buckypaper for full-range detection of human motions. Nanoscale 2018, 10, 14966-14975. [CrossRef] [PubMed]

4. Shi, G.; Zhao, Z.; Pai, J.H.; Lee, I.; Zhang, L.; Stevenson, C.; Ishara, K.; Zhang, R.; Zhu, H.; Ma, J. Highly sensitive, wearable, durable strain sensors and stretchable conductors using graphene/silicon rubber composites. Adv. Funct. Mater. 2016, 26, 7614-7625. [CrossRef]

5. Guan, L.; Nilghaz, A.; Su, B.; Jiang, L.; Cheng, W.; Shen, W. Stretchable-Fiber-Confined Wetting Conductive Liquids as Wearable Human Health Monitors. Adv. Funct. Mater. 2016, 26, 4511-4517. [CrossRef]

6. Yu, X.-G.; Li, Y.-Q.; Zhu, W.-B.; Huang, P.; Wang, T.-T.; Hu, N.; Fu, S.-Y. A wearable strain sensor based on a carbonized nano-sponge/silicone composite for human motion detection. Nanoscale 2017, 9, 6680-6685. [PubMed]

7. Li, X.; Yang, T.; Yang, Y.; Zhu, J.; Li, L.; Alam, F.E.; Li, X.; Wang, K.; Cheng, H.; Lin, C.T. Large-Area Ultrathin Graphene Films by Single-Step Marangoni Self-Assembly for Highly Sensitive Strain Sensing Application. Adv. Funct. Mater. 2016, 26, 1322-1329. [CrossRef]

8. Yang, T.; Wang, W.; Zhang, H.; Li, X.; Shi, J.; He, Y.; Zheng, Q.-S.; Li, Z.; Zhu, H. Tactile sensing system based on arrays of graphene woven microfabrics: Electromechanical behavior and electronic skin application. ACS Nano 2015, 9, 10867-10875. [CrossRef]

9. Moon, M.-W.; Cha, T.-G.; Lee, K.-R.; Vaziri, A.; Kim, H.-Y. Tilted Janus polymer pillars. Soft Matter 2010, 6, 3924-3929. [CrossRef]

10. Lee, S.W.; Park, J.J.; Park, B.H.; Mun, S.C.; Park, Y.T.; Liao, K.; Seo, T.S.; Hyun, W.J.; Park, O.O. Enhanced sensitivity of patterned graphene strain sensors used for monitoring subtle human body motions. ACS Appl. Mater. Interfaces 2017, 9, 11176-11183. [CrossRef] [PubMed]

11. Yao, S.; Zhu, Y. Wearable multifunctional sensors using printed stretchable conductors made of silver nanowires. Nanoscale 2014, 6, 2345-2352. [CrossRef]

12. Yamamoto, D.; Nakata, S.; Kanao, K.; Arie, T.; Akita, S.; Takei, K. A Planar, Multisensing Wearable Health Monitoring Device Integrated with Acceleration, Temperature, and Electrocardiogram Sensors. Adv. Mater. Technol. 2017, 2, 1700057. [CrossRef]

13. Ho, M.D.; Ling, Y.; Yap, L.W.; Wang, Y.; Dong, D.; Zhao, Y.; Cheng, W. Percolating Network of Ultrathin Gold Nanowires and Silver Nanowires toward "Invisible" Wearable Sensors for Detecting Emotional Expression and Apexcardiogram. Adv. Funct. Mater. 2017, 27, 1700845. [CrossRef]

14. Liang, B.; Lin, Z.; Chen, W.; He, Z.; Zhong, J.; Zhu, H.; Tang, Z.; Gui, X. Ultra-stretchable and highly sensitive strain sensor based on gradient structure carbon nanotubes. Nanoscale 2018, 10, 13599-13606. [CrossRef] [PubMed]

15. Wang, Y.; Wang, L.; Yang, T.; Li, X.; Zang, X.; Zhu, M.; Wang, K.; Wu, D.; Zhu, H. Wearable and highly sensitive graphene strain sensors for human motion monitoring. Adv. Funct. Mater. 2014, 24, 4666-4670. [CrossRef]

16. Choi, D.Y.; Kim, M.H.; Oh, Y.S.; Jung, S.-H.; Jung, J.H.; Sung, H.J.; Lee, H.W.; Lee, H.M. Highly Stretchable, Hysteresis-Free Ionic Liquid-Based Strain Sensor for Precise Human Motion Monitoring. ACS Appl. Mater. Interfaces 2017, 9, 1770-1780. [CrossRef]

17. Seyedin, S.; Razal, J.M.; Innis, P.C.; Jeiranikhameneh, A.; Beirne, S.; Wallace, G.G. Knitted strain sensor textiles of highly conductive all-polymeric fibers. ACS Appl. Mater. Interfaces 2015, 7, 21150-21158. [CrossRef] [PubMed]

18. Song, P.; Cao, Z.; Cai, Y.; Zhao, L.; Fang, Z.; Fu, S. Fabrication of exfoliated graphene-based polypropylene nanocomposites with enhanced mechanical and thermal properties. Polymer 2011, 52, 4001-4010. [CrossRef]

19. Cao, Z.; Song, P.; Fang, Z.; Xu, Y.; Zhang, Y.; Guo, Z. Physical wrapping of reduced graphene oxide sheets by polyethylene wax and its modification on the mechanical properties of polyethylene. J. Appl. Polym. Sci. 2012, 126, 1546-1555. [CrossRef]

20. Meng, F.; Wang, H.; Huang, F.; Guo, Y.; Wang, Z.; Hui, D.; Zhou, Z. Graphene-based microwave absorbing composites: A review and prospective. Compos. Part B Eng. 2018, 137, 260-277. [CrossRef]

21. Shi, G.; Araby, S.; Gibson, C.T.; Meng, Q.; Zhu, S.; Ma, J. Graphene Platelets and Their Polymer Composites: Fabrication, Structure, Properties, and Applications. Adv. Funct. Mater. 2018, 1706705. [CrossRef] 
22. Song, P.; Dai, J.; Chen, G.; Yu, Y.; Fang, Z.; Lei, W.; Fu, S.; Wang, H.; Chen, Z.-G. Bioinspired Design of Strong, Tough and Thermally Stable Polymeric Materials via Nanoconfinement. ACS Nano 2018, 12, 9266-9278. [CrossRef] [PubMed]

23. Ye, S.; Rathmell, A.R.; Chen, Z.; Stewart, I.E.; Wiley, B.J. Metal nanowire networks: The next generation of transparent conductors. Adv. Mater. 2014, 26, 6670-6687. [CrossRef] [PubMed]

24. Lee, H.; Song, C.; Baik, S.; Kim, D.; Hyeon, T.; Kim, D.-H. Device-assisted transdermal drug delivery. Adv. Drug Deliv. Rev. 2018, 127, 35-45. [CrossRef] [PubMed]

25. Godin, B.; Touitou, E. Transdermal skin delivery: Predictions for humans from in vivo, ex vivo and animal models. Adv. Drug Deliv. Rev. 2007, 59, 1152-1161. [CrossRef] [PubMed]

26. Merino, S.; Martín, C.; Kostarelos, K.; Prato, M.; Vázquez, E. Nanocomposite hydrogels: 3D polymer-nanoparticle synergies for on-demand drug delivery. Acs Nano 2015, 9, 4686-4697. [CrossRef] [PubMed]

27. Zaman, I.; Kuan, H.C.; Meng, Q.; Michelmore, A.; Kawashima, N.; Pitt, T.; Zhang, L.; Gouda, S.; Luong, L.; Ma, J. A facile approach to chemically modified graphene and its polymer nanocomposites. Adv. Funct. Mater. 2012, 22, 2735-2743. [CrossRef]

28. Kopecki, Z.; Ruzehaji, N.; Turner, C.; Iwata, H.; Ludwig, R.J.; Zillikens, D.; Murrell, D.F.; Cowin, A.J. Topically applied flightless I neutralizing antibodies improve healing of blistered skin in a murine model of epidermolysis bullosa acquisita. J. Investig. Dermatol. 2013, 133, 1008-1016. [CrossRef]

29. Haidari, H.; Zhang, Q.; Melville, E.; Kopecki, Z.; Song, Y.; Cowin, A.J.; Garg, S. Development of topical delivery systems for flightless neutralizing antibody. J. Pharm. Sci. 2017, 106, 1795-1804. [CrossRef]

30. Kopecki, Z.; Yang, G.N.; Arkell, R.M.; Jackson, J.E.; Melville, E.; Iwata, H.; Ludwig, R.J.; Zillikens, D.; Murrell, D.F.; Cowin, A.J. Flightless I over-expression impairs skin barrier development, function and recovery following skin blistering. J. Pathol. 2014, 232, 541-552. [CrossRef]

31. Aksak, B.; Murphy, M.P.; Sitti, M. Gecko inspired micro-fibrillar adhesives for wall climbing robots on micro/nanoscale rough surfaces. In Proceedings of the IEEE International Conference on Robotics and Automation, Pasadena, CA, USA, 19-23 May 2008; pp. 3058-3063.

32. Wang, D.-Y.; Tao, L.-Q.; Liu, Y.; Zhang, T.-Y.; Pang, Y.; Wang, Q.; Jiang, S.; Yang, Y.; Ren, T.-L. High performance flexible strain sensor based on self-locked overlapping graphene sheets. Nanoscale 2016, 8, 20090-20095. [CrossRef] [PubMed]

33. Wu, S.; Ladani, R.B.; Zhang, J.; Ghorbani, K.; Zhang, X.; Mouritz, A.P.; Kinloch, A.J.; Wang, C.H. Strain Sensors with Adjustable Sensitivity by Tailoring the Microstructure of Graphene Aerogel/PDMS Nanocomposites. ACS Appl. Mater. Interfaces 2016, 8, 24853-24861. [CrossRef]

34. Wang, S.; Xiao, P.; Liang, Y.; Zhang, J.; Huang, Y.; Wu, S.; Kuo, S.-W.; Chen, T. Network cracks-based wearable strain sensors for subtle and large strain detection of human motions. J. Mater. Chem. C 2018, 6, 5140-5147. [CrossRef]

35. Liu, H.; Li, Q.; Zhang, S.; Yin, R.; Liu, X.; He, Y.; Dai, K.; Shan, C.; Guo, J.; Liu, C. Electrically conductive polymer composites for smart flexible strain sensors: A critical review. J. Mater. Chem. C 2018, 6, 12121-12141. [CrossRef]

36. Venugopal, A.; Colombo, L.; Vogel, E. Contact resistance in few and multilayer graphene devices. Appl. Phys. Lett. 2010, 96, 013512. [CrossRef]

37. Amjadi, M.; Pichitpajongkit, A.; Lee, S.; Ryu, S.; Park, I. Highly stretchable and sensitive strain sensor based on silver nanowire-elastomer nanocomposite. ACS Nano 2014, 8, 5154-5163. [CrossRef] [PubMed]

38. Oh, S.; Kim, J.; Chang, S.T. Highly sensitive metal-grid strain sensors via water-based solution processing. RSC Adv. 2018, 8, 42153-42159. [CrossRef]

39. Souri, H.; Bhattacharyya, D. Highly sensitive, stretchable and wearable strain sensors using fragmented conductive cotton fabric. J. Mater. Chem. C 2018, 6, 10524-10531. [CrossRef]

40. Lee, Y.; Park, J.; Cho, S.; Shin, Y.-E.; Lee, H.; Kim, J.; Myoung, J.; Cho, S.; Kang, S.; Baig, C. Flexible ferroelectric sensors with ultrahigh pressure sensitivity and linear response over exceptionally broad pressure range. ACS Nano 2018, 12, 4045-4054. [CrossRef]

41. Pang, C.; Koo, J.H.; Nguyen, A.; Caves, J.M.; Kim, M.G.; Chortos, A.; Kim, K.; Wang, P.J.; Tok, J.B.H.; Bao, Z. Highly skin-conformal microhairy sensor for pulse signal amplification. Adv. Mater. 2015, 27, 634-640. [CrossRef]

42. Zhu, Z.; Li, R.; Pan, T. Imperceptible epidermal-iontronic interface for wearable sensing. Adv. Mater. 2018, 30, 1705122. [CrossRef] [PubMed] 
43. Dagdeviren, C.; Su, Y.; Joe, P.; Yona, R.; Liu, Y.; Kim, Y.-S.; Huang, Y.; Damadoran, A.R.; Xia, J.; Martin, L.W. Conformable amplified lead zirconate titanate sensors with enhanced piezoelectric response for cutaneous pressure monitoring. Nat. Commun. 2014, 5, 4496. [CrossRef] [PubMed]

44. Shah, D.S.; Middleton, C.; Gurdezi, S.; Horwitz, M.D.; Kedgley, A.E. The effects of wrist motion and hand orientation on muscle forces: A physiologic wrist simulator study. J. Biomech. 2017, 60, 232-237. [CrossRef] [PubMed]

45. Xu, H.; Lv, Y.; Qiu, D.; Zhou, Y.; Zeng, H.; Chu, Y. An ultra-stretchable, highly sensitive and biocompatible capacitive strain sensor from an ionic nanocomposite for on-skin monitoring. Nanoscale 2019, 11, 1570-1578. [CrossRef]

46. Huang, C.-M.; Chang, H.-C.; Kao, S.-T.; Li, T.-C.; Wei, C.-C.; Chen, C.; Liao, Y.-T.; Chen, F.-J. Radial pressure pulse and heart rate variability in heat-and cold-stressed humans. Evid.-Based Complement. Altern. Med. 2011, 2011. [CrossRef] [PubMed]

47. Shokawa, T.; Imazu, M.; Yamamoto, H.; Toyofuku, M.; Tasaki, N.; Okimoto, T.; Yamane, K.; Kohno, N. Pulse wave velocity predicts cardiovascular mortality. Circ. J. 2005, 69, 259-264. [CrossRef] [PubMed]

48. Wang, X.; Dong, L.; Zhang, H.; Yu, R.; Pan, C.; Wang, Z.L. Recent progress in electronic skin. Adv. Sci. 2015, 2, 1500169. [CrossRef] [PubMed]

49. Ranamukhaarachchi, S.; Lehnert, S.; Ranamukhaarachchi, S.; Sprenger, L.; Schneider, T.; Mansoor, I.; Rai, K.; Häfeli, U.; Stoeber, B. A micromechanical comparison of human and porcine skin before and after preservation by freezing for medical device development. Sci. Rep. 2016, 6, 32074. [CrossRef] [PubMed]

50. Proksch, E.; Brandner, J.M.; Jensen, J.M. The skin: An indispensable barrier. Exp. Dermatol. 2008, 17, 1063-1072. [CrossRef]

51. Duscher, D.; Neofytou, E.; Wong, V.W.; Maan, Z.N.; Rennert, R.C.; Inayathullah, M.; Januszyk, M.; Rodrigues, M.; Malkovskiy, A.V.; Whitmore, A.J. Transdermal deferoxamine prevents pressure-induced diabetic ulcers. Proc. Natl. Acad. Sci. USA 2015, 112, 94-99. [CrossRef]

52. Zhang, K.; Tang, X.; Zhang, J.; Lu, W.; Lin, X.; Zhang, Y.; Tian, B.; Yang, H.; He, H. PEG-PLGA copolymers: Their structure and structure-influenced drug delivery applications. J. Control. Release 2014, 183, 77-86. [CrossRef] [PubMed]

53. Nafee, N.; Taetz, S.; Schneider, M.; Schaefer, U.F.; Lehr, C.-M. Chitosan-coated PLGA nanoparticles for DNA/RNA delivery: Effect of the formulation parameters on complexation and transfection of antisense oligonucleotides. Nanomed. Nanotechnol. Biol. Med. 2007, 3, 173-183. [CrossRef] [PubMed]

54. Takeuchi, I.; Suzuki, T.; Makino, K. Skin permeability and transdermal delivery route of 50-nm indomethacin-loaded PLGA nanoparticles. Colloids Surf. B Biointerfaces 2017, 159, 312-317. [CrossRef] [PubMed]

55. Rassu, G.; Salis, A.; Porcu, E.P.; Giunchedi, P.; Roldo, M.; Gavini, E. Composite chitosan/alginate hydrogel for controlled release of deferoxamine: A system to potentially treat iron dysregulation diseases. Carbohydr. Polym. 2016, 136, 1338-1347. [CrossRef] [PubMed]

(C) 2019 by the authors. Licensee MDPI, Basel, Switzerland. This article is an open access article distributed under the terms and conditions of the Creative Commons Attribution (CC BY) license (http://creativecommons.org/licenses/by/4.0/). 